\section{IMPACT OF HEMISPHERIC DOMINANCE ON BEHAVIOURAL LATERALITY STYLE}

\author{
Reena Menezes, Dr. Priyanka Kacker, Prof. C.R. Mukundan \\ M.Sc. Neuropsychology, \\ Institute of Behavioural Science, \\ Gujarat Forensic Sciences University, Gandhinagar \\ Assistant Professor, \\ Institute of Behavioural Science, \\ Gujarat Forensic Sciences University, Gandhinagar \\ Axxonet Brain Research Laboratory, \\ Axxonet System Technologies, \\ Bangalore
}

\begin{abstract}
The cerebral hemispheres play crucial role in forming the mental state of human being. "Hemisity", is an aspect of the brain that considers two distinguished modes of information flow into the brain their differential processing modes within the two hemispheres. These two modes of information processing become the foundation for the development of differences in the functions of the two cerebral hemispheres. Each hemisphere has certain areas assigned with certain functions. The dominance of a particular processing mode in the brain of an individual gives rise to specific behavioural and personality characteristics. An individual may be excellent in performing certain tasks and may exhibit characteristics which would define his individuality, his identity and the dominant talent. These characteristics may be strongly correlated with the hemispheric dominance. Thus, it is considered that there may be a close link between hemisity and behavioural characteristics of an individual. This research aims at understanding the correlation between Hemisity and behavioural characteristics of a group of volunteers. The samples selected for this research were 150 males and 150 females of age group, 18-25 years. To measure characteristics exhibited by an individual Binary Preference Questionnaire (Morton, 2003) will be used and a questionnaire is developed and validated by an expert neuropsychologist to measure the brain dominance and characteristics exhibited by the right or left-brain oriented individuals. The results showed that less than half of the total number of samples reflected hemispheric dominance and exhibited respective hemisphere characteristics. However, more than half of the samples reflected hemispheric dominance but exhibited characteristics of nondominant hemisphere. The hypothesis that there exists a relation between Hemisity and behavioural characteristics exhibited by the right and left-brain oriented individuals is not completely supported by the data obtained. Thus, the result stands inconsistent with the data obtained and the earlier researches.
\end{abstract}

Key Words: Hemispheric Dominance, Brain Lateralization

\section{INTRODUCTION}

\section{HEMISITY}

The brain which seems to be an aggregate or a unified entity has been found to be bilateral. Basically the brain is divided into two hemispheres: the left hemisphere and the right hemisphere. These two hemispheres are connected to each other through tracts containing a massive bundle of fibres which are called Cerebral Commissures. The largest cerebral commissure connecting the two hemispheres is known as the Corpus Callosum. Its main function is transfer of learned information between the two hemispheres. The two hemispheres are not entirely symmetric. The most acquainted and familiar asymmetry in humans has been found to be language (Springer \& Deutsch, 1993). It was discovered long ago that damage to any one of the hemispheres of the brain would have a diminishing effect on the movement of the opposite side of the body. Researchers have always been posed with the question related to the existence of two hemispheres. Many of them have researched about the differentiating factor between the two hemispheres and process of interaction between them. Various structural and functional asymmetries were identified and studied and various Hemispheric interaction models were put forth. Recently, Positron Emission Tomography (PET) and Functional MRI (fMRI) were used and new hemispheric asymmetries were discovered. These studies have associated changes in the brain lateralization as a function of variables of population such as aging.

Certain functions are localized in specific brain areas. Thus, different areas of the brain have specialized functions. The localization of different functions to one side of the brain is termed as 


\section{GAP INDIAN JOURNAL OF FORENSICS AND BEHAVIOURAL SCIENCES}

( ISSN - 2582-8177)

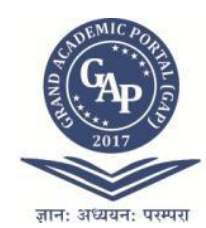

perspective that the right hemisphere had the potential of basic language processing but usually has grammatical abilities.

The Wada test, created by Juhn Wada at the Montreal Neurological Institute in 1949, was designed specifically to study lateralization. It is commonly used to determine the importance of the left or the right hemisphere in language. In this test, a barbiturate is injected in the carotid artery on one side and gradually transported to the opposite hemisphere. Either one of the hemispheres to suppress it for some time so that the functioning of individual hemisphere can be observed. Here, during this period of suppression of hemisphere, the capability to use language is tested. If the left hemisphere is anaesthetized and is the dominant hemisphere, the patient loses all ability to speak, whereas if the left hemisphere is anaesthetized but the right hemisphere is dominant, the patient will continue to speak throughout the procedure (Bear et al., 2007). A study published in 1977, Brenda Milner used the Wada test to demonstrate that $98 \%$ of right-handed people and $70 \%$ of left-handed people have a dominant left hemisphere with regards to language and speech function. The results depicted that $2 \%$ of right-handed people have a dominant right hemisphere, which is the same percentage of patients that exhibit aphasia following a lesion to the right hemisphere (Branch et al., 1964). This test is usually employed in medical situations like a neurosurgery, where the surgeons determine the dominant hemisphere in order to confirm that the removal of certain brain tissues will not interfere with the crucial functions of language and speech comprehension as well as processing.

Researches done earlier solely rested on the recording obtained from lateral eye movements. In Lateral eye movement studies, it was primarily assumed that the lateral eye movement in the right direction during cognitive tasks reflected dominant left hemisphere processing (Kinsbourne, 1972) as opposed to lateral eye movement in the left direction that indicated right hemisphere processing. It was consistently found that lateral eye movement in the right direction occurred in verbal tasks whereas lateral eye movement in the left direction were present in spatial tasks.

Despite the use of LEMs to evidence hemisphericity by a number of theorists, (Beaumont, Young \& McManus; Christie \& Raine, 1988; Springer 1985) the resulting studies into the validity of the technique have revealed inconsistent results. Lateral eye movements could not be proved as a valid indicator of hemispheric dominance due to lack of evidences due to which, the use of this technique has been criticized by Beaumont, Young and McManus (1984), a view supported by Raine (1991). New methods that facilitated the study of hemisphericity are electroencephalogram (EEG) and event-related potentials (ERP). However, various differences in cortical anatomy and lack of uniformity issues relating to electrode placement and data analysis have been put forth by theorists (Halasek \& Highberg, 2001). In addition to this, an argument stating the dependence of ERP studies on the number of times a trial should be repeated to create artificial linguistic processing conditions (Halasek \& Highberg, 2001) and the hindrance it caused to the potential of the experimenter to differentiate between perceptual, linguistic and cognitive factors. Use EEG and ERP studies of hemisphericity have been tagged as problematic due to the question on the ability of the experimenter to interpret such studies with key precision (Halasek \& Highberg).

Hemisphericity is a phenomenon in relation to the distinguishing factors between the left and the right hemispheres in thinking and behaviour. The concept of hemisphericity was surrounded by the belief that it was based on the rate of inclination or declination between the left and right hemisphere in terms of functional asymmetry. After several contradicting and striking reports, the field of hemisphericity finally collapsed in the 1980s (Beaumont, Young, and McManus, 1984). After about twenty five years of the suppression of the concept of hemisphericity, a new term called "hemisity" was introduced. This binary concept explained that an individual is innately either left or right brain oriented in thinking and behavioural styles. This fact is irreversible. Based on this binary concept, three independent, but highly intercorrelated biophysical methods (dichotic deafness, two-hand line bisection, two-hand mirror tracing) have shown persistent significant differences between individuals (Morton, 2001, 2002, 2003a,b,c). The measurement of the cross sectional area of the MRI midline corpus callosum contributed to the discovery of the first neuroanatomical difference in hemisity subtypes (Morton and Rafto, 2006). A concrete fact that threefold differences were present in corpus callosal information transfer capacity between right and left brain-oriented hemisity subtypes was found. Morton, in his study assessed 133 hemisity subtype subjects on the basis of the answers given by them to five behavioural questionnaires (Zenhausern, 1978; Morton, 2002, 2003c). He observed that the answers given by the hemisity subtype subjects to the "either-or" choices were opposite. In order to make interpretation easier, the items were grouped into five categories based on the grounds of priority which are as follows:

(1) Logical orientation

(2) Type of consciousness

(3) Fear level and Sensitivity

(4) Social-Professional Orientation

(5) Pair bonding-Spousal dominance style

Hemisity has helped to categorize people into a duality of anatomy and behaviour. This was made possible by use of three biophysical methods (Morton, 2001, 2002, 2003a,b). As the result of further extension of this research, hemisity can now be determined, based upon which side of an individual's vgACC is thickest, as revealed by a $3 \mathrm{~min}$ MRI scan (Morton and Rafto, 2010). What thinking and behavioural style differences exist 


\section{$\stackrel{000}{2}$ GRAND ACADEMIC PORTAL RESEARCH JOURNALS \\ GAP INDIAN JOURNAL OF FORENSICS AND BEHAVIOURAL SCIENCES ( ISSN - 2582-8177)}

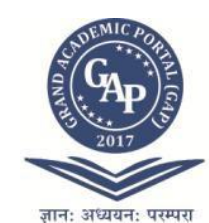

feelings. Studies of commisurotomized patients suggest that the dissociation between affect and cognition seen in alexithymia is related to a functional deconnection of the hemispheres (HoppeandBogen, 1977). A clinical study of a patient with a genesis of the corpus callosum revealed severe alexithymia (Buchananetal., 1980). Increased size of the corpus callosum may be associated with greater interhemispheric communication (Christman, 1995).Right hemisity is associated with a larger area of the corpus callosum(Morton and Rafto, 2006),and with lower levels of alexithymia. It is predicted that left hemisity will be associated with higher scores on the Toronto Alexithymia Scale (Bagbyetal.,1994).

Repressors: A repressive defence style, i.e., preferential use of the defence mechanism of repression has been found to be associated with relatively greater left hemisphere activation (Waldinger and Van Strien, 1995). It was associated with the retardation of the perception of threat and advocate self-esteem. It also showed a preference for defence mechanisms characterized by the inhibition of interpersonal conflict and ambivalent or negative emotions, and by selective accentuation of the positive (Schwartz, 1990). Tomarken and Davidson (1994). A higher left frontal EEG activation, an elevated autonomic responsivity and systolic blood pressure is significant in repressors. The repressive coping style may be associated with a functional hemispheric deconnection (Schwartz, 1990). Davidson (1984), discovered that there was relative deficiency in the transfer of negative affective information from one hemisphere to the other in repressors. Thus, the repressive style of coping seems to have a strong association with greater left frontal activation and reduction in the transfer of negative affective information across the corpus callosum. It is here predicted that left hemisity will be associated with a repressive style, as indicated by high scores on measures of social desirability, and low scores on measures of anxiety on the MCSDS (Tomarken and Davidson, 1994).

\section{FEAR LEVEL AND SENSITIVITY}

Anxiety-confidence: It seems that the left brain oriented individuals were more inclined to anxiety than the right brain oriented ones. Individuals with left hemisity do not prefer to discuss or talk about their emotions as opposed to those with right hemisity who are comfortable in discussing and experiencing emotions. Posttraumatic disorder may be associated with left hemisity along with other illnesses that are based on anxiety and caused by stress.

\section{SOCIAL AND PROFESSIONAL ORIENTATION}

The two important aspects of social and professional life are extroversion and introversion. Based on the research, an association was discovered between left brain oriented individuals and introversion. Similarly, right brain oriented individuals were associated with extroversion. Introversion is associated with greater cerebral blood flow while extroversion with less cerebral blood flow (Mathew et al., 1984). A non-verbal decoding deficit was observed in introverts which implied lesser functioning of right hemisphere in introverts (Lieberman and Rosenthal, 2001). Introverts exhibit hyper-anxious Type A behaviour in which they tend to suppress stimulation in order to control excessive baseline arousal (LudvighandHapp, 1974). They usually tend to medicate themselves with depressants and as a result are more inclined to alcoholism (Morton, 2011, 2012). On the other hand, the extroverts display a low baseline arousal for which they seek stimulation so that the arousal level can be balanced to an optimum level. Individuals with right hemisity seems to choose stimulating substances over depressants (Morton, 2011, 2012). The researches and studies quoted earlier have shown a strong association between higher baseline arousal and morningness, alexithymia and the repressive defence style, all of which coincidently are indicative of individuals with left brain orientation. Extraversion has been found to be significantly associated with various characteristics associated with transliminality and right hemisity, including creativity and mania/hypomania (Cassano et al., 2009). Extraversion is also associated with higher levels of emotional awareness (Igarashi et al., 2011).

Theory of mind abilities: The theory of mind is the capacity to draw a conclusion or make a deduction about the intentions, feelings and mental states of other individual and use this data foretell and control behaviour (Premack and Woodruff, 1978). Theory of mind abilities have been found to be associated with various aspects of non-literal language, including metaphor, humor, irony, and sarcasm (Langdon et al., 2002; Channon et al., 2005). Based on evidences right hemisity individuals are believed to have a greater potential to understand and know what others are thinking. Damage to the right hemisphere has shown to impair the comprehension of similes, metaphors, proverbs, sarcasm, humour, and other non-literal inferences (Brownell et al., 1990). Both affective prosody and sarcasm perception are thought to depend on the right hemisphere (Shamay-Tsoory et al., 2005). The right frontal lobe is involved to a great extent in the coordination between the theory of mind abilities and self-awareness. (Stuss et al., 2001). A right hemisphere stroke may cause impairment in the tasks associated with the theory of mind (Happé et al., 1999). 


\section{$\stackrel{000}{=}$ \\ GAP INDIAN JOURNAL OF FORENSICS AND BEHAVIOURAL SCIENCES}

GRAND ACADEMIC PORTAL RESEARCH JOURNALS ( ISSN - 2582-8177)

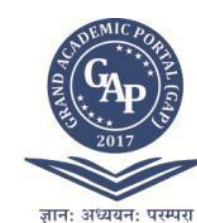

\section{PAIR BONDING AND SPOUSAL DOMINANCE STYLE}

\section{Attachment}

The items included in the category of Pair Bonding and Spousal Dominance style consider the behaviour in terms of attachment. The research implied that in individuals with left hemisity, the behavioural attachment system is activated at a very low level whereas in individuals with right hemisity, there is a greater activation of the behavioural attachment system. Theoretical arguments and empirical studies have suggested that attachment behaviours are mediated predominantly by the right hemisphere, particularly the right orbitofrontal cortex (Mohr et al., 2008). This research has predicted an inference that left hemisity is more closely associated with an avoidant spousal attachment style as opposed to right hemisity which has a strong inclination towards the dominant spousal attachment style.

It is not yet explicitly defined the extent to which the traits of hemisity assist the different aspects of personality. A further research to explore in depth the relation between the different traits of hemisity and the overall personality is the need of the hour.

Evidence has been amassed that the left cerebral hemisphere in most right- handed individuals manifests facilities for language (Broca, 1865), has an orientation for local detail (Robertson and Lamb, 1991), has object abstraction-identification abilities (Kosslyn, 1987), and appears to possess a hypothesis-generating, event "Interpreter" (Gazzaniga, 1989, 2000;Wolford et al., 2000). In contrast, the RH has been demonstrated to excel in global analysis (Robertson and Lamb, 1991; Proverbio et al., 1994), object localization (Kosslyn et al., 1989), facial recognition (Milner, 1968), and spatial con- struction (Sperry, 1968). Among the about $90 \%$ of humans who are right-handed (Coren, 1992), language is located in the LH in about 96\% of them (Knecht et al., 2000). Of the remaining about $10 \%$ of left handed individuals, some $73 \%$ of these also have language in their left cerebrum (Knecht et al., 2000). Thus, the conclusion drawn is that the Left hemisphere houses language ability in about $93.7 \%$ of us.

\section{BINARY QUESTIONNAIRE AND HEMISITY QUESTIONNAIRE:}

Recently the Binary Questionnaire and the Hemisity Questionnaires have also been developed and utilized (Morton, 2012).

Several MRI studies were performed to determine the structural differences of the brain of the left brain and right brain oriented individuals. One of the important neuroanatomical differences discovered was that the cross-sectional area of the midline of the corpus callosum of right brain oriented individuals was three times larger as compared to that of left brain oriented individuals and to be unrelated to sex or handedness (Morton and Rafto, 2006). The other fact discovered was that the bilateral anterior cingulate cortex (ACC) in areas 24 and 24' was upto 50\% larger on the right side for individuals with right brain orientation as compared to the individuals with left brain orientation it was up to 50\% larger on the left (Morton and Rafto,2010).

Once the hemisity of an individual could be determined, the next question that arose was whether there could be significant differences in the behavioural preferences of the right hemisity and left hemisity individuals? Morton attempted to draw a relation between the hemisity subtype of an individual and his behavioural preferences. Based on his research, he put forth the result that individuals with left brain orientation tend to be top-down, detail oriented and reason deductively whereas individuals with right brain orientation tend to be bottom up, big picture and prefer inductive reasoning. In terms of the Type of Consciousness, individuals with right brain orientation were more visual, relied on concrete reasoning and had the ability to find commonalities between objects. On the other hand, individuals with left brain orientation, were more verbal, relied on abstract reasoning and had a strong inclination to find differences between objects. In regards to Fear, Level and Sensitivity, the left brain oriented individuals were more sensitive, silent, emotion-avoiding and defensive whereas the right brain oriented individuals were bold, talkative and invasive. For Social and Professional Orientation, individuals with left hemisity were more independent, secluded and competitive in comparison to individuals with right hemisity who were more orderly, responsible and open. In relation to Pair bonding style and Spousal Dominance, individuals with left brain orientation were less dominant and demanded privacy, calmness, coolness, try to avoid emotions with logic and assist the spouse. Right brain orientated individuals were comparatively more dominant, demanded closeness from spouse and required to be restored of their confidence in the faithfulness of the spouse and support, along with being intuitive and directive.

Usually for most of the left brain oriented individuals, the executive element of the anterior cingulate lies in the hemisphere that contains language which implies transcallosal communication is not required which is opposite as compared to the scenario in which the executive element would be present in the contralateral hemisphere which does not house language. In such cases, the corpus callosum in the individuals with left brain orientation could be guessed to be smaller in comparison to those with right brain orientation. Several beliefs associating gender, laterality and brain surfaced in the past. Initially, when corpus callosum was studied, the aspect of hemisity remained aloof. This resulted in a confusion and the results were grouped based on sex or handedness with hemisity, a major factor influencing corpus callosum (Morton and Rafto, 2006). 


\section{0 GRAND ACADEMIC PORTAL RESEARCH JOURNALS

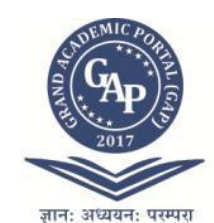

\section{SAMPLE:}

Total 250 students within the age group of 18-25 years were selected randomly from various colleges of Gandhinagar.

\section{VARIABLES:}

Independent Variables:

Hemisity (Left and right brain orientation) and behavioural characteristics

Dependent Variables:

The scores obtained on the Right-Left Questionnaire and the Binary Preference

Questionnaire (Morton, 2003).

\section{DIAGNOSTIC TOOLS:}

Right-Left Questions Questionnaire (V. Ramachandran, 1988)

The Right-Left Questions is a 17-item self-report measure of the hemispheric dominance. It is mainly useful for screening the hemispheric dominance of individuals. It appears to be a valid and reasonable measure of hemisity.

Binary Preference Questionnaire (Morton, 2003).

It is a 40 -item, report in which the items are grouped into four categories based on the grounds of priority which are as follows:

(1) Logical orientation

(2) Type of consciousness

(3) Fear level, Arousal and Sensitivity

(4) General Behavioural Style

It helps in identifying the characteristics exhibited by an individual under the influence of the dominant hemisphere. The internal validity and reliability of the questionnaire was assessed by Cronbach's alpha.

\section{PROCEDURE:}

The sample was randomly collected from various colleges in Gandhinagar. Students in the age group of 18-25 years were selected and given the questionnaires to be filled. The subjects were made comfortable and explained the purpose of research.

First they were asked to fill the Right-Left Questionnaire. Appropriate instructions were given. They were informed that the Questionnaire had 17 items, each having an option a and b. They had to choose either of the options and rate them on a scale of 1 to 10.1 being the lowest and 10 being the highest.

Next, they were asked to fill the Binary Preference Questionnaire. They were informed that the Questionnaire had 40 items, each having an option a and b. They had to choose either of the options that suited them the best. The Subjects were informed that there was no time limit. However, they were asked to fill the Questionnaires as soon as possible and avoid leaving any response blank.

Once the questionnaires were completed, they were collected back.

\section{RESULTS \& DISCUSSION}

The score of each sample on the Right-Left questions questionnaire and the Binary Preference questionnaire was calculated. The total number of samples that reflected hemispheric dominance along with specific behavioural characteristics confined to the respective hemispheres was calculated. The percentage of samples exhibiting such relation was calculated and represented using a Pie chart. Refer to Pie chart no. 1. PIE CHART NO. 1: Hemispheric Dominance and Behavioural Laterality style.

\section{HEMISPHERIC DOMINANCE AND BEHAVIOURAL LATERALITY STYLE}

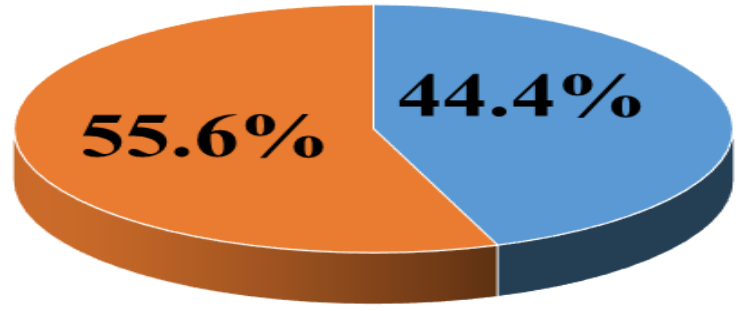

$\operatorname{match}=111$ 


\section{0 GRAND ACADEMIC PORTAL RESEARCH JOURNALS \\ GAP INDIAN JOURNAL \\ OF FORENSICS AND BEHAVIOURAL SCIENCES \\ ( ISSN - 2582-8177)}

Out of 250 samples , 111 samples i.e. 44.4\% reflected hemispheric dominance and exhibited respective hemisphere characteristics. Also, 139 samples i.e. 56.6\% reflected hemispheric dominance but exhibited characteristics of non-dominant hemisphere.

Next the total number of samples exhibiting Right hemispheric dominance and its characteristics was calculated. The percentage of samples exhibiting this dominance was computed. Similar calculation was performed for the samples exhibiting Left hemispheric dominance and its characteristics. The result was represented using a Pie chart. Refer to Pie chart no. 2.

\section{- Right hemisphere - Right-Left hemisphere \\ Left hemisphere}

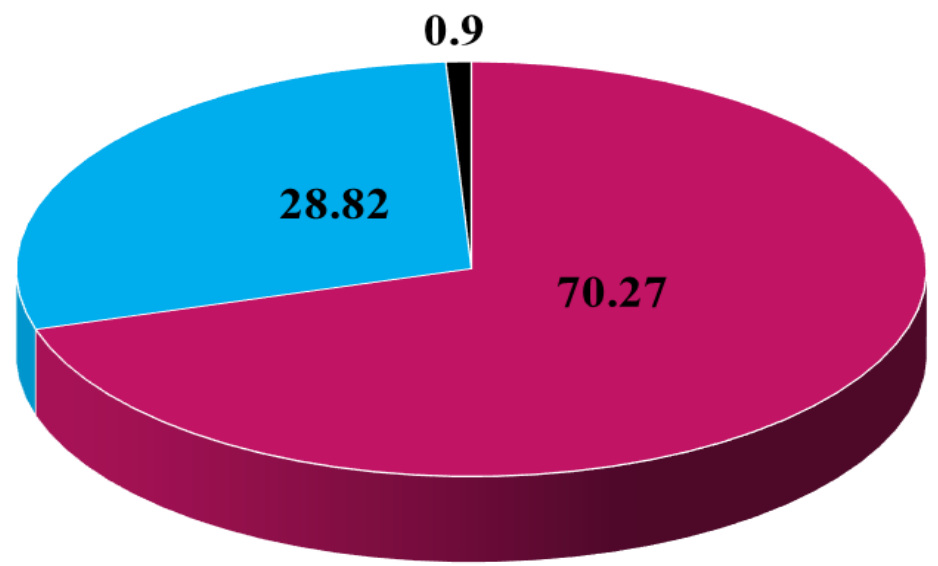

PIE CHART NO. 2: Hemispheric Dominance and Behavioural Characteristics.

Out of the 111 samples that exhibited hemispheric dominance and showed respective characteristics, 78 samples i.e. $70.27 \%$ were Right Hemisphere dominant and exhibited characteristics pertaining to the right hemisphere. On the other hand, 32 samples i.e. $28.82 \%$ were Left Hemisphere dominant and exhibited characteristics pertaining to the left hemisphere. However, 1 sample i.e. $0.9 \%$ was Right-Left hemisphere dominant and exhibited characteristics pertaining to both the right as well as the left hemisphere.

\section{CONCLUSION}

The hypothesis that there exists a relation between Hemisity and behavioural characteristics exhibited by the right and left-brain oriented individuals is not completely supported by the data obtained. Thus, the result stands inconsistent with the data obtained and the earlier researches.

\section{REFERENCES}

[1] Bruce E. Morton, S. E. (2010). Behavioural Laterality Advance: Neuroanatomical Evidence for the Existence of Hemisity. Personality and Individual Difference, 49. 34-42.

[2] Gulpinar, M. A. (2005). The Principles of Brain-Based Learning and Constructivist Models in Education. Education Sciences: Theory and Practice.

[3] Maartensson, F. (2007). Lateralization of Language Functions in the Human Brain. Neurolinguistics.

[4] Pinel, J. P. (2003). BioPsychology. Boston: Pearson Education, Inc.

[5] Sander Daselaar, R. C. (n.d.). Age-Related changes in Hemispheric Organization. Cognitive Neuroscience of Aging: Linking Cognitve and Cerebral Aging, 325-353. 